\title{
PERIODIC ORBITS OF PERIOD 3 IN THE DISC
}

\author{
BORIS KOLEV
}

\begin{abstract}
Let $f$ be an orientation preserving homeomorphism of the disc $D^{2}$ which possesses a periodic point of period 3 . Then either $f$ is isotopic, relative the periodic orbit, to a homeomorphism $g$ which is conjugate to a rotation by $2 \pi / 3$ or $4 \pi / 3$, or $J$ has a periodic point of least period $n$ for each $n \in \mathbb{N}^{*}$.
\end{abstract}

\section{INTRODUCTION}

Some very famous results of one dimensional dynamics state that a continuous map of the interval which has a periodic point of period 3 has periodic points of all other periods $[16,18]$ and positive topological entropy [3]. These results are certainly not true in higher dimensions; a rotation by $2 \pi / 3$ in the disc $D^{2}$ has periodic points of period 3 and 1 only and topological entropy zero. In fact the period alone is not enough to specify a periodic orbit of a surface's homeomorphism $f$ in order to get information about the structure of the set of periodic points of $f$.

What sort of stronger hypothesis do we have to add in order to get similar results in dimension two ? This problem has been extensively studied these last decades and a lot of results have emerged. Blanchard and Franks [1] have shown that an orientation reversing homeomorphism $f$ of the 2-sphere which has a point of period $p$ and a point of period $q$ where $p$ and $q$ are distinct odd integers has positive topological entropy. If in addition, $f$ is $C^{1+\epsilon}$ [15], $f$ has periodic points of arbitrary large period. Handel [12] has generalized this result to orientation reversing homeomorphisms of any compact surface $M$ using Nielsen-Thurston theory. Following an idea of Bowen [2], these authors specify a periodic point $z$ by the isotopy class of the restriction $f_{z}$ of $f$ to $M_{z}=M-o(z, f)$; the complement in $M$ of the orbit of $z$ under $f$. This idea has since been formalized by Boyland [4] who introduced the 'braid type' of a periodic orbit. Specifically, if $\left[f_{z}\right]$ denotes the isotopy class of $f_{z}$ in $M_{z}$, define the braid type of $o(z, f)$, denoted $b t(z, f)$, to be the conjugacy class of $\left[f_{z}\right]$ in the mapping class group of $M_{z}$. Recently several authors $[10,17]$ have characterized all the braid

Date: 28 January 1994.

1991 Mathematics Subject Classification. 54H20, 58F13, 34C35.

Key words and phrases. Dynamics of surfaces homeomorphisms; periodic orbits. 
types which appear for diffeomorphisms of genus zero surfaces with topological entropy zero.

More recently, Gambaudo, van Strien and Tresser [11] have given an example of a braid type of period 3 in the disc which imply all other periods and positive topological entropy, which is the exact analogue of the result in one dimensional dynamics stated previously. In this paper we show that this is a quite general result, namely:

Theorem 1.1. If $f$ is an orientation preserving homeomorphism of the disc $D^{2}$ which has a periodic point $z$ of period 3, then either $f$ is isotopic to a homeomorphism $g$ which is conjugate to a rotation by $2 \pi / 3$ or $4 \pi / 3$ or $f$ has periodic points of least period $n$ for each $n \in \mathbb{N}^{*}$.

In short terms, this result means that pseudo-Anosov 3 braid types of the disc imply all other periods. By the way, this result is obviously not true for the only two other 3-braid types which are not pseudoAnosov as cited previously. Therefore, it should be emphasized that Theorem 1.1 is the best possible extension of the paper. "Period three implies chaos" [16] for the class of interval maps to the class of orientation preserving homeomorphisms of the disc $D^{2}$.

The proof of this theorem is based on the structure of pseudo-Anosov homeomorphisms in the disc with three holes and upon some results of John Franks about the existence of periodic points for homeomorphisms of the annulus [9]. In section 2, we recall the basic facts we need and give the proof of Theorem 1.1 in section 3 .

\section{BACKGROUND AND DEFINITIONS}

2.1. Pseudo-Anosov homeomorphisms. We begin by recalling some properties of pseudo-Anosov homeomorphisms that we need and that can be found in [7] or [8] (see also [14] for the definition of the fixed point index). A pseudo-Anosov homeomorphism $\phi$ of a compact orientable manifold $M$ with negative Euler characteristic is a homeomorphism such that there exists a pair of transverse measured foliations $F^{u}$ and $\mathcal{F}^{s}$ and a number $\lambda>1$ with $\phi\left(\mathcal{F}^{u}\right)=\lambda^{-1} \mathcal{F}^{u}$ and $\phi\left(\mathcal{F}^{s}\right)=\lambda \mathcal{F}^{s}$.

2.1.1. Each of the foliation have a finite number of singularities and at least one on each component of the boundary of $M$. These singularities are one-prong singularities on the boundary and $p$-prongs singularities with $p \geq 3$ in the interior of $M . \mathcal{F}^{u}$ and $\mathcal{F}^{s}$ have the same singularities in the interior of $M$ and on each component of the boundary the singularities of $\mathcal{F}^{u}$ and $\mathcal{F}^{s}$ alternate. Moreover, we have the following formula for each foliation

$$
\sum_{s}\left(2-p_{s}\right)=2 \chi(M)
$$


where the sum is taken over all the singularities $s, p_{s}$ is the number of prongs at $s$ if $s$ lies in the interior of $M$ and $p_{s}=3$ if $s$ is a one-prong singularity on the boundary.

2.1.2. Let $x$ be a fixed point of $\phi^{n}$. Then $\operatorname{Ind}\left(x, \phi^{n}\right) \neq 0, \operatorname{Ind}\left(x, \phi^{n}\right) \leq$ 1 and $\operatorname{Ind}\left(x, \phi^{n}\right)<0$ if $x$ lies on the boundary of $M$.

2.1.3. Let $x$ be a fixed point of $\phi$.

- If $\operatorname{Ind}(x, \phi)=-1$, then $\operatorname{Ind}\left(x, \phi^{n}\right)=-1$ for all $n$.

- If $\operatorname{Ind}(x, \phi)=1$, then there exist two positive integers $p$ and $q$ such that:

$$
\operatorname{Ind}(x, \phi)=\operatorname{Ind}\left(x \cdot \phi^{2}\right)=\ldots=\operatorname{Ind}\left(x, \phi^{q-1}\right)=1
$$

and

$$
\operatorname{Ind}\left(x, \phi^{q}\right)=1-p .
$$

In that case, there are exactly $p$ stable (resp. unstable) halfleaves starting at $x$ and the permutation induced by $\phi$ on theses half-leaves consists of $p / q q$-cycles (if $p \geq 3, x$ is a singularity of the foliations).

2.1.4. If $\phi$ has a periodic point of least period $n$ which is not on the boundary of $M$, then every map $g$ isotopic to $\phi$ must possess a periodic point of least period $n$.

2.1.5. $\phi$ has a dense orbit.

2.2. Homeomorphisms of the annulus. Next, we briefly review some results about homeomorphisms of the annulus and chain transitivity [9]. Let $f: A \rightarrow A$ a homeomorphism of the annulus $A=$ $S^{1} \times[0,1]$ which is orientation preserving and boundary component preserving.Let $\pi: \tilde{A} \rightarrow A$ be the universal cover, $\tilde{A}=\mathbb{R} \times[0,1]$ and $p_{1}: \tilde{A} \rightarrow \mathbb{R}$ the projection onto the first coordinate.For each $x \in A$, choose a lift $\tilde{x} \in \tilde{A}$ and consider:

$$
\lim _{n \rightarrow \infty} \frac{p_{1}\left(\tilde{f}^{n}(\tilde{x})\right)-p_{1}(\tilde{x})}{n}
$$

where $\tilde{f}$ is a lift of $f$. When this limit exists, it is called the $\tilde{f}$-rotation number of $x$ and denoted $\rho(\tilde{f}, x)$. It is independent of the choice of the lift $\tilde{x}$ of $x$. If $x$ belongs to one component of the boundary, $\rho(x, \tilde{f})$ is defined and independent of the choice of $x$ on this component. Let $\rho_{1}(\tilde{f})$ and $\rho_{2}(\tilde{f})$ these two numbers. A periodic point $z$ is called a $p / q$ periodic point relative to $\tilde{f}$ if $z$ has least period $q$ and $\tilde{f}^{q}(\tilde{z})=\tilde{z}+(p, 0)$ for some lift (and hence all) $\tilde{z}$ of $z$.

A homeomorphism $f$ is called chain-transitive if for any $x, y \in A$ and $\epsilon>0$ there exists a sequence $x_{0}=x, x_{1}, \ldots, x_{n}=y, n \geq 1$ such that $d\left(f\left(x_{i}\right), x_{i+1}\right)<\epsilon$. In particular if $f$ has a dense orbit it is chain transitive. 
Theorem 2.1 (Franks). Let $f: A \rightarrow A$ be an orientation preserving, boundary component preserving homeomorphism which is chain transitive and $\tilde{f}: \tilde{A} \rightarrow \tilde{A}$ a lift of $f$. Then for every rational $p / q$ between $\rho_{1}(\tilde{f})$ and $\rho_{2}(\tilde{f})$, f has a $p / q$ periodic point.

\section{Proof of MAIN THEOREM}

We write $z_{i}=f^{i}(z),(i=1,2,3)$ for the points of the orbits of $z$ and set $o(z)=\left\{z_{1}, z_{2}, z_{3}\right\}$. Let $D_{z}=D^{2}-o(z), M$ be the compact surface obtained from $D_{z}$ by compactifying each puncture $z_{i}$ by a circle $S_{i}$ and $\pi: M \rightarrow D^{2}$ be the map which collapses each circle $S_{i}$ to $z_{i}$.

There is a natural isomorphism between the mapping class group of $D_{z}$ and the mapping class group of $M$. In our case $\left[f_{z}\right]$ is not reducible (in the sense of Nielsen-Thurston) because $z$ is a point of period 3 and there are no reducible braid type of prime period [4]. Consequently $\left[f_{z}\right]$ is either of finite order or of pseudo-Anosov type. If $\left[f_{z}\right]$ is of finite order, then there exists a homeomorphism $g$ in $D^{2}$ which is isotopic to $f$ rel. $o(z)$ and satisfy $g^{3}=I d$. Consequently, by a theorem of Kerékjártó $[5,6], g$ is conjugate to a rotation by $2 \pi / 3$ or $4 \pi / 3$ and we are done. If $\left[f_{z}\right]$ is of pseudo-Anosov type, we are going to show that $f$ possesses a periodic point of least period $n$ for each integer $n$. Let $\phi$ be a pseudo-Anosov homeomorphism of $M$ in the class $\left[f_{z}\right]$.

Lemma 3.1. $\phi$ has a periodic point of least period $n$ in the interior of $M$ for each $n \in \mathbb{N}^{*} \backslash\{3\}$.

Proof of Lemma 3.1. $\chi(M)=-2$ ( $M$ is a sphere with 4 holes !), so for each foliation $\mathcal{F}^{u}$ and $\mathcal{F}^{s}$ there is exactly one singularity on each boundary component of $M$ and no singularity in the interior of $M$ (2.1.1). Let $S_{\infty}$ be the component of $\partial M$ corresponding to the boundary of $D^{2}$. $S_{\infty}$ is invariant under $\phi$ and the singularities of the two foliations on $S_{\infty}$ are fixed points for $\phi$ (see Figure 1).

Let $L(\phi)$ be the Lefschetz number of $\phi$ (see [14] for definition and computation of the Lefschetz number). We have:

$$
L(\phi)=\sum_{x \in \operatorname{Fix}(\phi)} \operatorname{Ind}(x, \phi)=1-\operatorname{Tr}\left(\phi_{1}^{*}\right)=1,
$$

where $\phi_{1}^{*}$ is the action induced by $\phi$ on the first homology group of $M$. Therefore, by (2.1.2), $\phi$ has a fixed point $z_{0}$ such that $\operatorname{Ind}\left(z_{0}, \phi\right)=1$. It results from (2.1.2) that $z_{0}$ lies in the interior of $M$ and thus is not a singularity of the foliations. For the same reason $\left(L\left(\phi^{2}\right)=1\right), \phi^{2}$ has a a fixed point of index 1 in the interior of $M$ which is not a fixed point of $\phi$ (2.1.3). Therefore $\phi$ has a periodic point of period 2 in the interior of $M$.

Blow up $z_{0}$ into a circle $S_{0}$ (there is no problem to extend the pseudoAnosov $\phi$ to $S_{0}$ ) and collapse the three circles $S_{1}, S_{2}, S_{3}$ to the original 


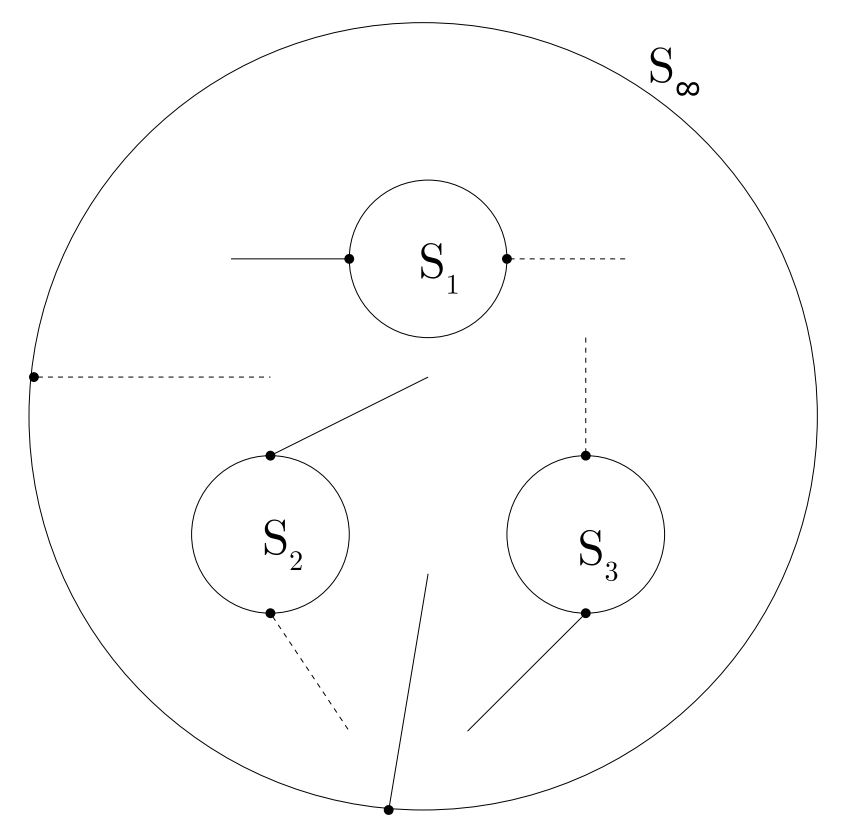

Figure 1. The manifold $M$

points $z_{1}, z_{2}, z_{3}$. Using the fact that $\phi$ has a dense orbit (2.1.5), we obtain by this way a chain-transitive homeomorphism of a closed annulus $A$ that we shall call $\Phi$.

Because $z_{0}$ is not a singularity of the foliation, we have $\operatorname{Ind}\left(z_{0}, \phi^{2}\right)=$ -1 by (2.1.3) and thus $\Phi$ has two period 2 periodic orbits on $S_{0}$.

Let $\tilde{\Phi}$ be any lift of $\Phi$ to the universal cover $\tilde{A}$ of $A$ and call $\rho_{0}(\tilde{\Phi})$ (resp. $\left.\rho_{\infty}(\tilde{\Phi})\right)$ the $\tilde{\Phi}$-rotation number of $\Phi / s_{0}\left(\operatorname{resp} . \Phi / s_{\infty}\right)$. Since $\Phi$ has a fixed point on $S_{\infty}$ and a periodic point of. period 2 on $S_{0}$, $\rho_{\infty}(\tilde{\Phi})=k$ for some $k \in \mathbb{Z}$ and $\rho_{0}(\tilde{\Phi})=(2 l+1) / 2$ for some $l \in \mathbb{Z}$. Using Theorem 2.1 we conclude that $\Phi$ has a periodic point of least period $n$ for each $n \geq 2$. All the periodic points of period $n \geq 3$ belong to the interior of $A$ and correspond therefore to periodic points of $\phi$.

Let us now give the proof of Theorem 1.1. Suppose first that $f$ is continuously differentiable around the points of o $(z)$. Then we can extend $f_{z}$ to a homeomorphism $F: M \rightarrow M$ which is isotopic to $\phi$ and there is a one to one correspondence between periodic points of $F$ in $\operatorname{int}(M)$ and periodic points of $f_{\mathrm{Z}}$ in $\operatorname{int}\left(D_{z}\right)$. Using the previous lemma and (2.1.4) $f$ has a periodic point of least period $n$ for every $n \in \mathbb{N}^{*}$.

In the case where $f$ is not supposed to be well-behaved near o $(z)$, we construct a sequence $f_{k}$ of homeomorphisms of $D^{2}$ which coincide with $f$ away from a $1 / k$-neighbourhood of $\mathrm{o}(z)$, which act on $\left\{z_{1}, z_{2}, z_{3}\right\}$ as $f$ but which are continuously differentiable around these points. Each 
$f_{k}$ extends to a homeomorphism $F_{k}$ of $M$ which is isotopic to $\phi$ in $M$ and $[13]$ :

Lemma 3.2 (Handel). For every $\epsilon>0$, there exists $\delta>0$ (independent of $k$ ) such that if $\phi$ has a periodic point $x$ of least period $n$ and $d(o(\phi, x), \partial M)>\epsilon$ then for every $k, F_{k}$ has a periodic point $x_{k}$ of least period $n$ such that $d\left(o\left(F_{k}, x_{k}\right), \partial M\right)>\delta$.

Therefore, $f$ has a periodic point of every period.

Remark. If we consider no longer a periodic point of period 3 but a periodic point of period $q>3$ such that $b t(z, f)$ is of type pseudoAnosov and if the singularities of the foliations of a pseudo-Anosov representative $\phi$ on $S_{\infty}$ are fixed points of $\phi$, then the proof of Theorem 1.1 holds. There exists a fixed point $z_{0}$ of index 1 for $\phi$ and such that when we blow up $z_{0}$ into a circle $S_{0}$ we get $\rho\left(\phi_{/ S_{0}}\right)=k / l$ where $k$ and $l$ are coprime with $2 \leq f \leq q-2$. Therefore, we can prove that $f$ has periodic points of period $n$ for every $n \geq q-2$. The general situation seems to be much more complex.

\section{REFERENCES}

[1] P Blanchard and J Franks, The dynamical complexity of orientation-reversing homeomorphisms of surfaces, Invent. Math. 62 (1980/81), no. 2, 333-339. MR MR595592 (82f:58073)

[2] R Bowen, Entropy and the fundamental group, The structure of attractors in dynamical systems (Proc. Conf., North Dakota State Univ., Fargo, N.D., 1977), Lecture Notes in Math., vol. 668, Springer, Berlin, 1978, pp. 21-29. MR MR518545 (80d:58049)

[3] R Bowen and J Franks, The periodic points of maps of the disk and the interval, Topology 15 (1976), no. 4, 337-342. MR MR0431282 (55 \#4283)

[4] P Boyland, Topological methods in surface dynamics, Topology Appl. 58 (1994), no. 3, 223-298. MR MR1288300 (95h:57016)

[5] A Constantin and B Kolev, The theorem of Kerékjártó on periodic homeomorphisms of the disc and the sphere, Enseign. Math. (2) 40 (1994), no. 3-4, 193-204. MR MR1309126 (95j:55005)

[6] S. Eilenberg, Sur les transformations periodiques de la surface de la sphère, Fund. Math. 22 (1934), 28-44.

[7] A Fathi and F Laudenbach, The dynamics of the lift of a pseudo-Anoxov diffeomorphism to the Poincaré disc, in Papadopoulos Thesis, Orsay, 1983.

[8] A Fathi, F Laudenbach, and V Poénaru, Travaux de Thurston sur les surfaces, Asterisque, vol. 66-67, SMF, 1979.

[9] J Franks, Recurrence and fixed points of surface homeomorphisms, Ergodic Theory Dynam. Systems 8* (1988), no. Charles Conley Memorial Issue, 99107. MR MR967632 (90d:58124)

[10] JM. Gambaudo, S van Strien, and C Tresser, The periodic orbit structure of orientation preserving diffeomorphisms on $D^{2}$ with topological entropy zero, Ann. Inst. H. Poincaré Phys. Théor. 50 (1989), no. 3, 335-356. MR MR1017968 (90j:57008)

[11] JM Gambaudo, S van Strien, and C Tresser, Vers un ordre de Sarkovskiu pour les plongements du disque préservant l'orientation, C. R. Acad. Sci. Paris Sér. I Math. 310 (1990), no. 5, 291-294. MR MR1042866 (91f:58072) 
[12] M Handel, The entropy of orientation reversing homeomorphisms of surfaces, Topology 21 (1982), no. 3, 291-296. MR MR649760 (84f:58097)

[13] - The rotation set of a homeomorphism of the annulus is closed, Comm. Math. Phys. 127 (1990), no. 2, 339-349. MR MR1037109 (91a:58102)

[14] BJ Jiang, Lectures on Nielsen fixed point theory, Contemporary Mathematics, vol. 14, American Mathematical Society, Providence, R.I., 1983. MR MR685755 (84f:55002)

[15] A Katok, Lyapunov exponents, entropy and periodic orbits for diffeomorphisms, Inst. Hautes Études Sci. Publ. Math. (1980), no. 51, 137-173. MR MR573822 (81i:28022)

[16] TY Li and JA. Yorke, Period three implies chaos, Amer. Math. Monthly 82 (1975), no. 10, 985-992. MR MR0385028 (52 \#5898)

[17] J Llibre and RS MacKay, A classification of braid types for diffeomorphisms of surfaces of genus zero with topological entropy zero, J. London Math. Soc. (2) 42 (1990), no. 3, 562-576. MR MR1087229 (92a:57019)

[18] OM Sarkovskii, Co-existence of cycles of a continuous mapping of the line into itself, Ukrain. Mat. Z̆. 16 (1964), 61-71. MR MR0159905 (28 \#3121)

CMi, 39 rue F. Joliot-Curie, 13453 Marseille cedex 13, France

E-mail address: kolev@cmi.univ-mrs.fr 